\title{
STATISTICAL PHRASE EXTRACTION AND INDEXING FOR MUSIC RETRIEVAL
}

\author{
Atsuhiro Takasu ${ }^{1}$, Teruhito Kanazawa ${ }^{2}$, and Jun Adachi ${ }^{1}$ \\ 1 National Institute of Informatics \\ 2 University of Tokyo (currently with KYA Group Corp.) \\ $\{$ takasu,tkana,adachi\}@nii.ac.jp
}

\begin{abstract}
This paper proposes a method for extracting phrases from songs in MIDI format and an indexing method for music retrieval. The proposed overall method accelerates query processing in two ways, by: (1) filtering songs by sub-sequences of notes, and (2) reducing the length of sequences in DP matching by using phrases instead of songs. We show experimentally that the proposed overall method reduces processing time to about $1 / 11$ with $10 \%$ loss of retrieval accuracy compared with a retrieval method that does not use either the phrase information or the index.
\end{abstract}

Keywords: Music Retrieval, Syntactical Analysis, Hidden Markov Model

\section{Introduction}

Music retrieval is a key function for music entertainment. Recently, more and more songs have been accumulated in digital formats such as Musical Instrument Digital Interface (MIDI), and effective and efficient music retrieval from large music databases will be required in the near future.

Textual metadata such as the composer's name and singer's name have been used frequently since the early stages of music retrieval. However, metadata construction is labor-intensive work and users often want to retrieve music in more direct ways such as through humming, singing or performing part of the music piece. Ghias used humming as the query mechanism and proposed a music retrieval system from an audio database[3]. Emotive words such as beautiful and clear (e.g., [8]) can form another type of query for music retrieval. Each piece of music is assigned to predefined emotive words and the query also consists of emotive words.

The original version of this chapter was revised: The copyright line was incorrect. This has been corrected. The Erratum to this chapter is available at DOI: 10.1007/978-0-387-35660-0_65 
The matching process varies depending on the query formulation. With textual metadata and emotive words, textual information retrieval methods can be applied. When using parts of musical pieces as in queries by humming, singing or performing, we must define similarities between melodies. In these approaches, melodies are represented using note sequences, and existing approximate matching techniques are applied to find pieces of music in the database similar to the query. Ghias [3] and Bakhmutova [2] reported the application of approximate matching to music retrieval. Another approach is to construct a feature space and to map both musical pieces and queries to points in the feature space. In this approach, the similarity is defined as the Euclidean distance or cosine measure between points in the feature space. Tsuji et al. [6] proposed a feature space consisting of trigram patterns in melodies. In this method, nine kinds of trigram pitch patterns were used as features and the feature value was defined as the frequency of appearance of the pattern in a musical piece.

We have been developing a music retrieval system. The goals of this system are:

- to identify structures that are useful for musical information retrieval,

- to develop a method for extracting these structures automatically, and

- to develop an indexing method for fast retrieval from large music databases.

In this system, we use musical phrases as fundamental structural elements. A phrase is a natural concept representing a musically meaningful component. We believe a phrase-based system has two advantages. Firstly, it accelerates query processing. A user generally gives a query as a phrase or a few phrases. By segmenting songs in the database into phrases, we can focus the matching procedure to related phrases, and consequently reduce the query processing time. This is the main subject of this paper. Secondly, we can use phrases as concise representations for musical retrieval. This paper proposes a phrase extraction method and applies it to indexing for music retrieval.

\section{Phrase Extraction}

In our music retrieval system, songs are stored in a database as MIDI data. Index "words" are extracted by

- extracting phrases from the song, and 
- selecting fragments of note sequences from segmented phrases.

On the other hand, a query by humming is first converted to MIDI data, then a set of index words is extracted from the MIDI data for selecting a set of candidate songs in the database. Finally the candidate songs are ranked based on edit distances and given to a user. The user selects songs from the listed result and listens to a representative phrase of the music until the target one is found. In this section we briefly describe a phrase extraction procedure and propose an efficient music retrieval method using the phrases in the next section.

Phrase extraction plays a key role in this system. Phrases are extracted from a MIDI song in the following steps:

1 segmenting a song to submelodies,

2 clustering submelodies,

3 detecting prefix clusters, and

4 correcting incorrectly extracted phrases by syntactical analysis.

These steps are described more fully below.

Segmentation. We use rests as delimiters of phrases. A fragment segmented by rests is referred to as a submelody. A phrase consists of a sequence of submelodies. Because MIDI data do not contain rest information explicitly, we extract submelodies by scanning the note sequence in the MIDI melody track, and then putting a rest where the duration between consecutive notes is greater than a fixed period of time. These fragments usually oversegment a song, and therefore they are merged into phrases in the following steps.

Clustering Submelodies. Submelodies are merged using the following characteristics:

- rule1: phrases are often repeated in a song, and

- rule2: repeated phrases have similar melody contours.

To apply these rules to phrase extraction, we must find phrases with similar melody contours. For this purpose, we first find the submelodies that are prefixes of phrases. Other submelodies in the song should be similar to these submelodies, by rule one. To find similar submelodies, a similarity measure based on the edit distance is used.

Let us first define the relative pitch sequence. For a pitch sequence $p_{1} p_{2} \cdots p_{n}$, the relative pitch sequence is $r_{1} r_{2} \cdots r_{n-1}$, where $r_{i}$ is 1,0 
or -1 if $p_{i+1}$ is higher than, equal to, or less than $p_{i}$, respectively. The relative pitch sequence is an approximate pitch sequence, and similarity is defined as the edit distance between relative pitch sequences. The decomposed submelodies are clustered into $G_{1}, G_{2}, \cdots, G_{k}$ according to their similarities.

Because the similarity distribution varies in each song, we require a procedure for adaptive thresholding. The similarity of pitch patterns of submelodies tends to have a bimodal distribution. We assume the similarity distribution is a mixture of two normal distributions

$$
\lambda N\left(\mu_{1}, \sigma_{1}^{2}\right)+(1-\lambda) N\left(\mu_{2}, \sigma_{2}^{2}\right)
$$

where $N\left(\mu, \sigma^{2}\right)$ stands for a normal distribution with mean $\mu$, and variance $\sigma^{2}$. The parameters $\mu_{1}, \mu_{2}, \sigma_{1}, \sigma_{2}$, and $\lambda$ are obtained by maximum likelihood estimation using the similarities of each pair of submelodies in a song. The similarity that has the minimum value in the estimated mixture distribution is used as the threshold for submelody clustering.

Prefix Cluster Detection. Submelodies of each group with a size of more than one are candidates for the prefix of a phrase. As the prefixes of phrases, we use submelodies in those groups that contain:

- the first submelody in the song;

- the submelody that follows a rest of length greater than or equal to four beats.

Syntactical Analysis. At this stage we obtain a phrase sequence $\mathbf{v} \equiv v_{1} v_{2} \cdots v_{l}$ where each phrase $v_{i}$ is a note sequence of MIDI data. Usually, songs consist of A melody, B melody, theme melody, and so on, and they are located regularly. By using the regularity of phrase sequences, we expect that the phrase extraction accuracy will be improved.

Let a set $C$ of categories of phrases be A melody, B melody, theme melody, and so on, and consider the problem of finding the most likely category sequence $\mathbf{c} \in C^{*}$ from phrase sequence $\mathbf{v}$. This problem is formalized as

$$
\underset{\mathbf{c} \in C^{*}}{\arg \max } P(\mathbf{c} \mid \mathbf{v})=\underset{\mathbf{c} \in C^{*}}{\arg \max } \frac{P(\mathbf{c}) P(\mathbf{v} \mid \mathbf{c})}{P(\mathbf{v})}=\underset{\mathbf{c} \in C^{*}}{\arg \max } P(\mathbf{c}) P(\mathbf{v} \mid \mathbf{c})
$$

The optimal category sequence may merge some of the automatically extracted phrases into a real phrase. In this way, oversegmentation of phrases is corrected by syntactical analysis, and consequently, accuracy may be improved. 
To solve (2) we apply the following steps:

1 construct a feature vector from each phrase represented with MIDI data, using average values of pitch, duration, and velocity of the submelody, and the cluster size to which the submelody belongs,

2 quantize the feature space according to the relationship between feature vectors and categories using decision trees for submelodies, and

3 apply an error-correcting parser using syntactical patterns of category sequence represented by a hidden Markov model.

See [5] for details.

\section{Music Retrieval}

The main research issue in query by humming is the measurement of similarity between the query and songs in the database. There are two kinds of errors in query by humming. One is caused by the user, that is, the hummed phrase differs from the object melody more or less, depending on the musical skill of the user. The other is caused by the audio recognition software that converts analog humming data into a MIDI note sequence. Therefore, music retrieval systems require robust similarity measurement ability.

To match erroneous sequences such as recognized humming with songs in the database, various approximate matching techniques can be applied. However, these techniques usually require a scan of all songs in the database, and therefore we require a technique to boost the approximate string matching for music retrieval from large databases (e.g., $[7,4])$.

Our system adopts two-pass retrieval by:

1 selecting candidate phrases from the database quickly using an inverted file, and

2 applying phrase-based similarity measurement based on the edit distance.

Let us consider a relative pitch sequence $\mathbf{p}$. Then, the selectivity of $\mathbf{p}$ for a music database is defined as

$\frac{\text { number of phrases with a relative pitch sequence containing } \mathbf{p}}{\text { number of phrases in the database }}$.

For a given selectivity $s$, a relative pitch sequence $r_{1} r_{2} \cdots r_{n}$ that has selectivity less than $s$ while the selectivity of $r_{1} r_{2} \cdots r_{n-1}$ is more than 
$s$, is called a "sequence of selectivity $s$ " and is used as an index sequence. An inverted file is constructed from such index sequences.

On the other hand, for the relative pitch sequence of a query, a set of suffixes $\left\{\mathbf{q}_{1}, \mathbf{q}_{2}, \cdots, \mathbf{q}_{n}\right\}$ is selected. For each suffix $\mathbf{q}_{i}$, we can collect phrases that contain the prefix of $\mathbf{q}_{i}$ of selectivity $s$ efficiently using the inverted file. By taking the intersection and/or union of phrases corresponding to the suffixes, we can construct candidate phrase sets. It is clear that intersection produces fewer candidates, although it is less robust as regards errors in the query sequences, while union has the reverse characteristics. By combining the union and intersection of suffixes, as well as selecting the number of suffixes, we can design a filter flexibly depending on the accuracy of the query.

For the selected phrases, we measure the similarity based on the edit distance. For a relative pitch sequence $\mathbf{q}$ of a query, and a relative pitch sequence $\mathbf{p}$ of a phrase, the similarity of $\mathbf{p}$ and $\mathbf{q}$ is defined as

$$
\operatorname{Sim}_{p}(\mathbf{p}, \mathbf{q}) \equiv \min _{1 \leq i \leq j} E D\left(\mathbf{q}, \mathbf{p}_{i}\right)
$$

where $\mathbf{p}_{i}$ stands for the suffix starting from the $i$-th character of $\mathbf{p}$, and $E D$ stands for the edit distance. The same similarity on sequences of durations of notes, denoted as $\operatorname{Sim}_{d}(\mathbf{p}, \mathbf{q})$, is calculated, and finally, the similarity of a query and a phrase is defined as the linear combination of these two similarities with some weight coefficient $w$, i.e.,

$$
\operatorname{Sim}(\mathbf{p}, \mathbf{q}) \equiv w \operatorname{Sim}_{p}(\mathbf{p}, \mathbf{q})+(1-w) \operatorname{Sim}_{d}(\mathbf{q}, \mathbf{p}) .
$$

\section{Experimental Results}

We applied the present method to the retrieval of Japanese popular music. The database consists of 645 Japanese popular songs in MIDI format. These songs contain a total of 5772 phrases. Phrases were extracted and an inverted file was constructed from the database. A test dataset of 66 queries was given by three volunteers in our laboratory. These queries were converted to MIDI note sequences by a commercial audio-to-MIDI program [1], then candidate phrases were selected, and finally, they were ranked according to similarity (3).

In this experiment, we chose three suffixes for each query and constructed filters as unions of them for selectivities of $0.01,0.02,0.04$, $0.06,0.08$ and 0.1 . The program was implemented in Java 1.4 and executed on a personal computer with a Pentium III $850 \mathrm{MHz} \mathrm{CPU}$ and $512 \mathrm{MB}$ main memory. Figure 1a compares the accuracy of filtered and non-filtered music retrieval, where accuracy means the fraction of times 


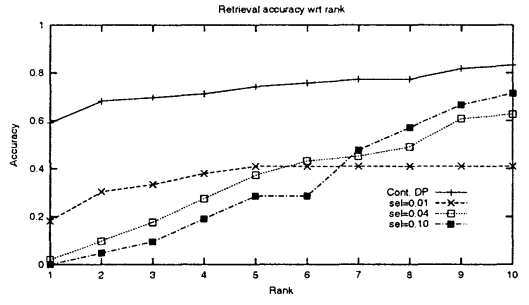

Figure 1a. Retrieval accuracy with respect to rank.

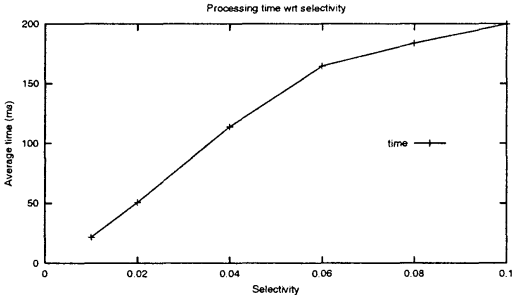

Figure 1 b. Processing time with respect to selectivity.

that the object song is included in the top $n$-ranked retrieval results. In the graph, "Cont. DP" stands for the accuracy without filtering and "sel=0.1", "sel=0.04" and "0.01" stand for the accuracy achieved with filter of selectivity $0.1,0.04$ and 0.01 , respectively. This graph shows that filters of lower selectivity tend to have higher accuracy for high-ranked retrieval results, however have lower accuracy for low-ranked retrieval results. This means that a filter of low selectivity tends to filter out the correct song.

Figure $1 \mathrm{~b}$ depicts the average query processing time for each selectivity. It shows that processing time is almost linear with the selectivity, i.e., the number of candidate phrases. When applying query processing without using a filter and phrases, the average query processing time was $2173.6 \mathrm{~ms}$, that is, it took about 11 times as long as the case of filtering with selectivity 0.1 .

The proposed method improves processing time in two ways. First, filtering reduces the number of songs to be processed, i.e., the number of disk accesses. This characteristic is very important for large databases. Second, phrase-based similarity measurement reduces the length of sequences compared in the DP matching, and consequently, reduces the processing time. In this example, the difference between the proposed method and non-filtered matching comes from the second effect, because the song database is small enough to be held in memory. Figures 1a and $1 \mathrm{~b}$ show that the presented method reduces the processing time to about $1 / 11$, with $10 \%$ loss of accuracy for retrieval results ranked in the top 10 .

\section{Conclusion}

In this paper, we proposed a method for extracting phrases from songs in MIDI format, and an indexing method for music retrieval. We conducted an experiment using 645 Japanese popular songs and showed 
that the proposed overall method reduces processing time to about $1 / 11$ with $10 \%$ loss of retrieval accuracy compared with a retrieval method that does not use phrase information or the index. In the experiment, we used similarity based on the edit distance. However, the proposed method will be effective for other similarity measures using dynamic programming. By tuning the parameters of the index, we will be able to improve the retrieval accuracy. Parameter tuning is a future problem.

\section{References}

[1] Wildcat Canyon Software: Autoscore http://www.wildcat.com/Site/Homepage.htm

[2] I.V. Bakhmutova, V.D. Gusev and T.N. Titkova, "The Search for Adaptations in Song Melodies", Computer Music Journal, Vol. 21, No.1, pp. 58-67, 1997.

[3] A. Ghias, J. Logan, D. Chamberlin and B.C. Smith, "Query By Humming - Musical Information Retrieval in an Audio Database", In Proc. of ACM Multimedia '95, 1995.

[4] N. Kosugi, Y. Nishihara, S. Kon'ya, M. Yamamuro and K. Kushima, "Music Retrieval by Humming - Using Similarity Retrieval over High Dimensional Feature Vector Space", Proc. of IEEE Pacific Rim Conference on Communications, Computers and Signal Processing, pp. 404-407, 1999.

[5] A. Takasu, T. Kanazawa, T. Yanase and J. Adachi, "Music Structure Analysis and Its Application to Theme Phrase Extraction", Lecture Notes in Computer Science 1696, pp. 92-105, 1999.

[6] Y. Tsuji, M. Hoshi, and T. Ohmori, "Local Patterns of a Melody and Its Applications to Retrieval by Sensitive Words (in Japanese)", Technical Report of IEICE, SP96 (124), pp. 17-24, 1997.

[7] S. Wu, and U. Manber, "Fast Text Searching Allowing Errors", Communications of $A C M$, Vol. 35, No. 10, pp. 83-91, 1996.

[8] T. Yoshino, H. Takagi, Y. Kiyoki, and T. Kitagawa, "An Automatic Metadata Creation Method for Music Data and its Application to Semantic Associative Search (in Japanese)", IPSJ SIG Notes, 98 (58), pp.109-116, 1998. 